\title{
EXISTENCE AND UNIQUENESS RESULTS FOR NONLINEAR IMPLICIT CAPUTO FRACTIONAL $q$-DIFFERENCE EQUATIONS WITH NONLOCAL CONDITIONS
}

\author{
ADEL LACHOURI ${ }^{1}$, ABDELOUAHEB ARDJOUNI $^{2, *}$, AHCENE DJOUDI $^{1}$ \\ ${ }^{1}$ Department of Mathematics, University of Annaba, Algeria \\ ${ }^{2}$ Department of Mathematics and Informatics, University of Souk Ahras, Algeria \\ *Corresponding author: abd_ardjouni@yahoo.fr
}

Received Aug. 28, 2020

\begin{abstract}
Авsтract. In this paper, we use the fixed point theory to obtain the existence and uniqueness of solutions for nonlinear implicit Caputo fractional $q$-difference equations with nonlocal conditions. An example is given to illustrate this work.
\end{abstract}

2010 Mathematics Subject Classification. 26A33; 39A13.

Key words and phrases. fractional $q$-difference equations; Caputo fractional $q$-difference derivatives; fixed point theorems; existence; uniqueness.

\section{InTRODUCTION}

Fractional differential equations without and with delay arise from a variety of applications including in various fields of science and engineering. In particular, problems concerning qualitative analysis of nonlinear and linear fractional differential equations without and with delay have received the attention of many authors, see [5]- [11], [15], [19]- [21], [25], [28] and the references therein.

The $q$-difference calculus or quantum calculus is an old subject that was initially developed by Jackson $[16,17]$. Basic definitions and properties of $q$-difference calculus can be found in the book mentioned in [18]. The problems of nonlinear fractional $q$-difference equations have aroused considerable attention. Many people pay attention to the existence and multiplicity of solutions or positive solutions for problems of nonlinear fractional $q$-difference equations, see [1]- [4], [12]- [14], [16], [17], [25], [22], [26], [27].

DOI: 10.28924/APJM/7-34

(2020 Asia Pacific Journal of Mathematics 
In [14], Ferreira investigate the existence of nontrivial solutions to the nonlinear $q$-fractional boundary value problem

$$
\left\{\begin{array}{l}
D_{q}^{\alpha} u(t)=-f(t, u(t)), 0 \leq t \leq 1, \\
u(0)=u(1)=0
\end{array}\right.
$$

where $0<q<1, D_{q}^{\alpha}$ denotes the Riemann-Liouville fractional $q$-derivative of order $1<\alpha \leq 2$ and $f:[0,1] \times \mathbb{R} \rightarrow \mathbb{R}$ is a given nonnegative continuous function.

In [26], Yang study the existence and uniqueness results for anti-periodic boundary value problems involving nonlinear fractional $q$-difference equations given by

$$
\left\{\begin{array}{l}
{ }^{C} D_{q}^{\alpha} u(t)=f(t, u(t)), 0 \leq t \leq 1, \\
u(0)=-u(1), D_{q} u(0)=-D_{q} u(1),
\end{array}\right.
$$

where $0<q<1,{ }^{C} D_{q}^{\alpha}$ denotes the Caputo fractional $q$-derivative of order $1<\alpha \leq 2$ and $f:[0,1] \times \mathbb{R} \rightarrow \mathbb{R}$ is a given continuous function.

In [1], the authors investigated the existence, uniqueness and Ulam stability of solutions for the following implicit fractional $q$-difference equation

$$
\left\{\begin{array}{l}
{ }^{C} D_{q}^{\alpha} u(t)=f\left(t, u(t),{ }^{C} D_{q}^{\alpha} u(t)\right), 0 \leq t \leq T, \\
u(0)=u_{0} \in \mathbb{R},
\end{array}\right.
$$

where $0<q<1,0<\alpha \leq 1, T>0, f:[0, T] \times \mathbb{R} \times \mathbb{R} \rightarrow \mathbb{R}$ is a given continuous function.

Inspired and motivated by the above works, we study the existence and uniqueness of solutions for the following implicit fractional $q$-difference equation with nonlocal conditions

$$
\left\{\begin{array}{l}
{ }^{C} D_{q}^{\alpha} u(t)=f\left(t, u(t),{ }^{C} D_{q}^{\alpha} u(t)\right), 0 \leq t \leq T, \\
u(0)+g(u)=u_{0} \in \mathbb{R},
\end{array}\right.
$$

where $0<q<1,0<\alpha \leq 1, T>0, f:[0, T] \times \mathbb{R} \times \mathbb{R} \rightarrow \mathbb{R}$ and $g: C([0, T], \mathbb{R}) \rightarrow \mathbb{R}$ are given continuous functions. In passing, we note that the application of nonlocal condition $u(0)+g(u)=u_{0}$ in physical problems yields better effect than the initial condition $u(0)=u_{0}$, see [10]. To show the existence and uniqueness of solutions, we transform (1.1) into an integral equation and then use the Krasnoselskii and Banach fixed point theorems.

The rest of this paper is organized as follows. In Section 2 we introduce some notations and lemmas, and state some preliminaries results needed in later sections. In Section 3, we prove the existence and uniqueness of solutions for the problem (1.1). Finally, an example is given in Section 4 to illustrate our results. 


\section{Preliminaries}

In this section, we recall some basic definitions and necessary lemmas related to fractional q-calculus and fixed point theorems that will be used throughout this paper.

Let $J=[0, T]$. By $C(J, \mathbb{R})$ we denote the Banach space of all continuous functions from $J$ into $\mathbb{R}$ with the norm

$$
\|u\|=\sup _{t \in J}|u(t)| .
$$

As usual $L^{1}(J)$ denotes the space of measurable functions $u: J \rightarrow \mathbb{R}$ which are Lebesgue integrable with the norm

$$
\|u\|_{1}=\int_{J}|u(t)| d t
$$

Let us recall some definitions and properties of fractional $q$-calculus. For $a \in \mathbb{R}$, we set

$$
[a]_{q}=\frac{1-q^{a}}{1-q} .
$$

The $q$ analogue of the power $(a-b)^{n}$ is

$$
(a-b)^{(0)}=1, \quad(a-b)^{(n)}=\prod_{k=0}^{n-1}\left(a-b q^{k}\right), a, b \in \mathbb{R}, n \in \mathbb{N} .
$$

In general,

$$
(a-b)^{(\alpha)}=a^{\alpha} \prod_{k=0}^{\infty}\left(\frac{a-b q^{k}}{a-b q^{k+\alpha}}\right), a, b, \alpha \in \mathbb{R} .
$$

Definition 2.1 ( [18]). The q-gamma function is defined by

$$
\Gamma_{q}(\xi)=\frac{(1-q)^{(\xi-1)}}{(1-q)^{\xi-1}}, \xi \in \mathbb{R} \backslash\{0,-1,-2, \ldots\} .
$$

Notice that the $q$-gamma function satisfies $\Gamma_{q}(1+\xi)=[\xi]_{q} \Gamma_{q}(\xi)$.

Definition 2.2 ( [18]). The q-derivative of order $n \in \mathbb{N}$ of a function $u: J \rightarrow \mathbb{R}$ is defined by $D_{q}^{0} u(t)=u(t)$,

$$
D_{q} u(t)=D_{q}^{1} u(t)=\frac{u(t)-u(q t)}{(1-q) t}, t \neq 0, D_{q} u(0)=\lim _{t \rightarrow 0} D_{q} u(t),
$$

and

$$
D_{q}^{n} u(t)=D_{q} D_{q}^{n-1} u(t), t \in J, n \in\{1,2, \ldots\}
$$

Set $J_{t}=\left\{t q^{n}: n \in \mathbb{N}\right\} \cup\{0\}$. 
Definition 2.3 ( [18]). The q-integral of a function $u: J_{t} \rightarrow \mathbb{R}$ is defined by

$$
I_{q} u(t)=\int_{0}^{t} u(s) d_{q} s=\sum_{n=0}^{\infty} t(1-q) q^{n} f\left(t q^{n}\right),
$$

provided that the series converges.

We note that $D_{q} I_{q} u(t)=u(t)$, while $u$ is continuous at 0 , then

$$
I_{q} D_{q} u(t)=u(t)-u(0) .
$$

Definition 2.4 ( [2]). The Riemann-Liouville fractional $q$-integral of order $\alpha \in \mathbb{R}^{+}$of a function $u: J \rightarrow \mathbb{R}$ is defined by $I_{q}^{0} u(t)=u(t)$ and

$$
I_{q}^{\alpha} u(t)=\int_{0}^{t} \frac{(t-q s)^{(\alpha-1)}}{\Gamma_{q}(\alpha)} u(s) d_{q} s, t \in J .
$$

Lemma $2.1([22])$. For $\alpha \in \mathbb{R}^{+}$and $\lambda \in(1, \infty)$ we have

$$
I_{q}^{\alpha}(t-a)^{(\lambda)}=\frac{\Gamma_{q}(1+\lambda)}{\Gamma_{q}(1+\lambda+\alpha)}(t-a)^{(\lambda+\alpha)}, 0<a<t .
$$

In particular

$$
I_{q}^{\alpha} 1=\frac{1}{\Gamma_{q}(1+\alpha)} t^{(\alpha)}
$$

Definition 2.5 ( [23]). The Riemann-Liouville fractional q-derivative of order $\alpha \in \mathbb{R}^{+}$of a function $u: J \rightarrow \mathbb{R}$ is defined by $D_{q}^{0} u(t)=u(t)$ and

$$
D_{q}^{\alpha} u(t)=D_{q}^{[\alpha]} I_{q}^{[\alpha]-\alpha} u(t), t \in J
$$

where $[\alpha]$ is the integer part of $\alpha$.

Definition 2.6 ( [23]). The Caputo fractional q-derivative of order $\alpha \in \mathbb{R}^{+}$of a function $u: J \rightarrow \mathbb{R}$ is defined by ${ }^{C} D_{q}^{0} u(t)=u(t)$, and

$$
{ }^{C} D_{q}^{\alpha} u(t)=I_{q}^{[\alpha]-\alpha} D_{q}^{[\alpha]} u(t), t \in J
$$

Lemma 2.2 ( [23]). Let $\alpha \in \mathbb{R}^{+}$. Then the following equality holds

$$
I_{q}^{\alpha C} D_{q}^{\alpha} u(t)=u(t)-\sum_{k=0}^{[\alpha]-1} \frac{t^{k}}{\Gamma_{q}(1+k)} D_{q}^{\alpha} u(0) .
$$

In particular, if $\alpha \in(0,1)$, then

$$
I_{q}^{\alpha C} D_{q}^{\alpha} u(t)=u(t)-u(0)
$$


Lastly in this section, we state the fixed point theorems which enable us to prove the existence and uniqueness of a solution of (1.1).

Theorem 2.1 (Banach's fixed point theorem [24]). Let $\Omega$ be a non-empty closed subset of a Banach space $(S,\|\|$.$) , then any contraction mapping \Phi$ of $\Omega$ into itself has a unique fixed point.

Theorem 2.2 (Krasnoselskii's fixed point theorem [24]). Let $\Omega$ be a non-empty bounded closed convex subset of a Banach space $(S,\|\|$.$) . Suppose that F_{1}$ and $F_{2}$ map $\Omega$ into $S$ such that

(i) $F_{1} u+F_{2} v \in \Omega$ for all $u, v \in \Omega$,

(ii) $F_{1}$ is continuous and compact,

(iii) $F_{2}$ is a contraction.

Then there is a $u \in \Omega$ with $F_{1} u+F_{2} u=u$.

\section{EXISTENCE AND UNIQUENESS}

Let us start by defining what we mean by a solution of the problem (1.1).

Definition 3.1. A function $u \in C^{1}(J, \mathbb{R})$ is said to be a solution of problem (1.1) if $u$ satisfies ${ }^{C} D_{q}^{\alpha} u(t)=f\left(t, u(t),{ }^{C} D_{q}^{\alpha} u(t)\right)$ for any $t \in J$ and $u(0)+g(u)=u_{0}$.

For the existence of solutions for the problem (1.1), we need the following auxiliary lemma.

Lemma 3.1. Let $u \in C(J, \mathbb{R})$ and $u^{\prime}$ exists, then $u$ is a solution of the initial value problem (1.1) if and only if it is a solution of the integral equation

$$
u(t)=u_{0}-g(u)+\int_{0}^{t} \frac{(t-q s)^{(\alpha-1)}}{\Gamma_{q}(\alpha)} f\left(s, u(s),{ }^{C} D_{q}^{\alpha} u(s)\right) d_{q} s .
$$

Proof. Suppose $u$ satisfies the problem (1.1). First we write (1.1) as

$$
I_{q}^{\alpha C} D_{q}^{\alpha} u(t)=I_{q}^{\alpha} f\left(t, x(t),{ }^{C} D_{0^{+}}^{\alpha} x(t)\right) .
$$

In view of Lemma 2.2, we have

$$
u(t)-u(0)=\int_{0}^{t} \frac{(t-q s)^{(\alpha-1)}}{\Gamma_{q}(\alpha)} f\left(s, u(s),{ }^{C} D_{q}^{\alpha} u(s)\right) d_{q} s .
$$

The condition $u(0)+g(u)=u_{0}$ implies that

$$
u(t)=u_{0}-g(u)+\int_{0}^{t} \frac{(t-q s)^{(\alpha-1)}}{\Gamma_{q}(\alpha)} f\left(s, u(s),{ }^{C} D_{q}^{\alpha} u(s)\right) d_{q} s .
$$

Then, we obtain (3.1). Since each step is reversible, the converse follows easily. This completes the proof. 
In the following subsections we prove existence, as well as existence and uniqueness results for the problem (1.1) by using a variety of fixed point theorems.

The following assumptions will be used in our main results

(H1) There exist constants $L_{1} \in \mathbb{R}^{+}$and $L_{2} \in(0,1)$ such that

$$
\left|f\left(t, u_{1}, v_{1}\right)-f\left(t, u_{2}, v_{2}\right)\right| \leq L_{1}\left|u_{1}-u_{2}\right|+L_{2}\left|v_{1}-v_{2}\right|
$$

for $t \in J$ and $u_{1}, u_{2}, v_{1}, v_{2} \in \mathbb{R}$.

(H2) There exists a constant $L_{g} \in(0,1)$ such that

$$
|g(u)-g(v)| \leq L_{g}\|u-v\|
$$

for $t \in J$ and $u, v \in C(J, \mathbb{R})$.

\subsection{Existence and uniqueness results via Banach's fixed point theorem.}

Theorem 3.1. Assume that the assumptions (H1)-(H2) are satisfied. If

$$
L_{g}+\frac{L_{1} T^{\alpha}}{\left(1-L_{2}\right) \Gamma_{q}(\alpha+1)}<1
$$

Then there exists a unique solution for the problem (1.1) on J.

Proof. We define the operator $\Phi: C(J, \mathbb{R}) \rightarrow C(J, \mathbb{R})$ by

$$
(\Phi u)(t)=u_{0}-g(u)+\int_{0}^{t} \frac{(t-q s)^{(\alpha-1)}}{\Gamma_{q}(\alpha)} h_{u}(s) d_{q} s,
$$

where $h_{u} \in C(J, \mathbb{R})$ be such that

$$
h_{u}(t)=f\left(t, u(t), h_{u}(t)\right)
$$

Clearly, the fixed points of operator $\Phi$ are solutions of problem (1.1).

For any $u, v \in C(J, \mathbb{R})$ and $t \in J$, we have

$$
\begin{aligned}
& |(\Phi u)(t)-(\Phi v)(t)| \\
& \leq|g(u)-g(v)|+\int_{0}^{t} \frac{(t-q s)^{(\alpha-1)}}{\Gamma_{q}(\alpha)}\left|h_{u}(s)-h_{v}(s)\right| d_{q} s \\
& \leq L_{g}\|u-v\|+\int_{0}^{t} \frac{(t-q s)^{(\alpha-1)}}{\Gamma_{q}(\alpha)}\left|h_{u}(s)-h_{v}(s)\right| d_{q} s
\end{aligned}
$$

where $h_{v} \in C(J, \mathbb{R})$ be such that

$$
h_{v}(t)=f\left(t, v(t), h_{v}(t)\right)
$$


By $(\mathrm{H} 1)$, we have

$$
\begin{aligned}
\left|h_{u}(t)-h_{v}(t)\right| & =\left|f\left(t, u(t), h_{u}(t)\right)-f\left(t, v(t), h_{v}(t)\right)\right| \\
& \leq L_{1}|u(t)-v(t)|+L_{2}\left|h_{u}(t)-h_{v}(t)\right| \\
& \leq \frac{L_{1}}{1-L_{2}}|u(t)-v(t)| .
\end{aligned}
$$

By replacing (3.4) in the inequality (3.3), we get

$$
\begin{aligned}
& |(\Phi u)(t)-(\Phi v)(t)| \\
& \leq L_{g}\|u-v\|+\frac{L_{1}}{1-L_{2}} \int_{0}^{t} \frac{(t-q s)^{(\alpha-1)}}{\Gamma_{q}(\alpha)}|u(t)-v(t)| d_{q} s \\
& \leq\left(L_{g}+\frac{L_{1} T^{\alpha}}{\left(1-L_{2}\right) \Gamma_{q}(\alpha+1)}\right)\|u-v\| .
\end{aligned}
$$

Therefore

$$
\|\Phi u-\Phi v\| \leq\left(L_{g}+\frac{L_{1} T^{\alpha}}{\left(1-L_{2}\right) \Gamma_{q}(\alpha+1)}\right)\|u-v\| .
$$

From (3.2), $\Phi$ is a contraction. As a consequence of Banach's fixed point theorem, we get that $\Phi$ has a unique fixed point which is a unique solution of the problem (1.1) on $J$.

\subsection{Existence results via Krasnoselskii's fixed point theorem.}

Theorem 3.2. Assume (H2) and the following hypothesis

(H3) There exist functions $p_{i} \in C\left(J, \mathbb{R}^{+}\right), i=1, \ldots, 3$ with $p_{3}^{*}=\sup _{t \in J} p_{3}(t)<1$ such that

$$
|f(t, u, v)| \leq p_{1}(t)+p_{2}(t)|u|+p_{3}(t)|v|, \text { for each } t \in J \text { and } u, v \in \mathbb{R} \text {. }
$$

If

$$
\frac{p_{2}^{*} T^{\alpha}}{\left(1-L_{g}\right)\left(1-p_{3}^{*}\right) \Gamma_{q}(\alpha+1)}<1,
$$

where $p_{2}^{*}=\sup _{t \in J} p_{2}(t)$. Then the boundary value problem (1.1) has at least one solution.

Proof. Let us fix

$$
\rho \geq R \Lambda,
$$

where

$$
R=\frac{\left(1-p_{3}^{*}\right) \Gamma_{q}(\alpha+1)}{\left(1-L_{g}\right)\left(1-p_{3}^{*}\right) \Gamma_{q}(\alpha+1)-p_{2}^{*} T^{\alpha}}
$$

and

$$
\Lambda=\left|u_{0}\right|+|g(0)|+\frac{p_{1}^{*}}{1-p_{3}^{*}} \frac{T^{\alpha}}{\Gamma_{q}(\alpha+1)},
$$


with $p_{1}^{*}=\sup _{t \in J} p_{1}(t)$. Consider the non-empty bounded closed convex subset

$\Omega=\{u \in C(J, \mathbb{R}),\|u\| \leq \rho\}$, and define two operators $F_{1}$ and $F_{2}$ on $\Omega$ as follows

$$
\left(F_{1} u\right)(t)=\int_{0}^{t} \frac{(t-q s)^{(\alpha-1)}}{\Gamma_{q}(\alpha)} h_{u}(s) d_{q} s
$$

and

$$
\left(F_{2} u\right)(t)=u_{0}-g(u) .
$$

We shall use the Krasnoselskii fixed point theorem to prove there exists at least one fixed point of the operator $F_{1}+F_{2}$ in $\Omega$. The proof will be given in several steps.

Step 1. We prove that $F_{1} u+F_{2} v \in \Omega$ for all $u, v \in \Omega$.

For any $u, v \in \Omega$, we have

$$
\begin{aligned}
& \left|\left(F_{1} u\right)(t)+\left(F_{2} v\right)(t)\right| \\
& \leq\left|u_{0}\right|+|g(v)|+\int_{0}^{t} \frac{(t-q s)^{(\alpha-1)}}{\Gamma_{q}(\alpha)}\left|h_{u}(s)\right| d_{q} s .
\end{aligned}
$$

By (H2) and (H3), we have

$$
\begin{aligned}
|g(v)| & \leq|g(v)-g(0)|+|g(0)| \\
& \leq L_{g}\|v\|+|g(0)| \\
& \leq L_{g} \rho+|g(0)|,
\end{aligned}
$$

and

$$
\begin{aligned}
\left|h_{u}(t)\right| & =\left|f\left(t, u(t), h_{u}(t)\right)\right| \\
& \leq p_{1}(t)+p_{2}(t)|u(t)|+p_{3}(t)\left|h_{u}(t)\right| \\
& \leq \frac{p_{1}^{*}}{1-p_{3}^{*}}+\frac{p_{2}^{*}}{1-p_{3}^{*}}|u(t)| .
\end{aligned}
$$

By replacing (3.6) and (3.7) in the inequality (3.5), we get

$$
\begin{aligned}
& \left|\left(F_{1} u\right)(t)+\left(F_{2} v\right)(t)\right| \\
& \leq\left|u_{0}\right|+L_{g} \rho+|g(0)|+\left(\frac{p_{1}^{*}}{1-p_{3}^{*}}+\frac{p_{2}^{*}}{1-p_{3}^{*}} \rho\right) \frac{T^{\alpha}}{\Gamma_{q}(\alpha+1)} .
\end{aligned}
$$

Thus

$$
\begin{aligned}
\left\|F_{1} u+F_{2} v\right\| & \leq \Lambda+\left(L_{g}+\frac{p_{2}^{*}}{1-p_{3}^{*}} \frac{T^{\alpha}}{\Gamma_{q}(\alpha+1)}\right) \rho \\
& \leq \frac{\rho}{R}+\left(1-\frac{1}{R}\right) \rho=\rho .
\end{aligned}
$$


Hence, $F_{1} u+F_{2} v \in \Omega$, for all $u, v \in \Omega$.

Step 2. We prove that $F_{1}$ is compact.

For all $u \in \Omega$ and by (3.7), we have

$$
\begin{aligned}
\left|\left(F_{1} u\right)(t)\right| & \leq \int_{0}^{t} \frac{(t-q s)^{(\alpha-1)}}{\Gamma_{q}(\alpha)}\left|h_{u}(s)\right| d_{q} s \\
& \leq\left(\frac{p_{1}^{*}}{1-p_{3}^{*}}+\frac{p_{2}^{*}}{1-p_{3}^{*}} \rho\right) \frac{T^{\alpha}}{\Gamma_{q}(\alpha+1)},
\end{aligned}
$$

thus

$$
\left\|F_{1} u\right\| \leq\left(\frac{p_{1}^{*}}{1-p_{3}^{*}}+\frac{p_{2}^{*}}{1-p_{3}^{*}} \rho\right) \frac{T^{\alpha}}{\Gamma_{q}(\alpha+1)} .
$$

Hence, $F_{1}$ is uniformly bounded on $\Omega$.

It remains to show that $F_{1}(\Omega)$ is equicontinuous, let $x \in \Omega$, then for any $0<t_{1}<t_{2} \leq T$ and from (3.7), we have

$$
\begin{aligned}
& \left|\left(F_{1} u\right)\left(t_{2}\right)-\left(F_{1} u\right)\left(t_{1}\right)\right| \\
& =\left|\int_{0}^{t_{2}} \frac{\left(t_{2}-q s\right)^{(\alpha-1)}}{\Gamma_{q}(\alpha)} h_{u}(s) d_{q} s \tau-\int_{0}^{t_{1}} \frac{\left(t_{1}-q s\right)^{(\alpha-1)}}{\Gamma_{q}(\alpha)} h_{u}(s) d_{q} s\right| \\
& \leq \frac{1}{\Gamma_{q}(\alpha)} \int_{0}^{t_{1}}\left|\left(t_{2}-q s\right)^{\alpha-1}-\left(t_{1}-q s\right)^{\alpha-1}\right|\left|h_{u}(s)\right| d_{q} s \\
& +\frac{1}{\Gamma_{q}(\alpha)} \int_{t_{1}}^{t_{2}}\left(t_{2}-q s\right)^{\alpha-1}\left|h_{u}(s)\right| d_{q} s \\
& \leq \frac{1}{\Gamma_{q}(\alpha)}\left(\frac{p_{1}^{*}}{1-p_{3}^{*}}+\frac{p_{2}^{*}}{1-p_{3}^{*}} \rho\right) \int_{0}^{t_{1}}\left(t_{1}-q s\right)^{\alpha-1}-\left(t_{2}-q s\right)^{\alpha-1} d_{q} s \\
& +\frac{1}{\Gamma_{q}(\alpha)}\left(\frac{p_{1}^{*}}{1-p_{3}^{*}}+\frac{p_{2}^{*}}{1-p_{3}^{*}} \rho\right) \int_{t_{1}}^{t_{2}}\left(t_{2}-q s\right)^{\alpha-1} d_{q} s \\
& \leq \frac{1}{\Gamma_{q}(\alpha+1)}\left(\frac{p_{1}^{*}}{1-p_{3}^{*}}+\frac{p_{2}^{*}}{1-p_{3}^{*}} \rho\right)\left(2\left(t_{2}-t_{1}\right)^{\alpha}+t_{1}^{\alpha}-t_{2}^{\alpha}\right) \\
& \leq \frac{2}{\Gamma_{q}(\alpha+1)}\left(\frac{p_{1}^{*}}{1-p_{3}^{*}}+\frac{p_{2}^{*}}{1-p_{3}^{*}} \rho\right)\left(t_{2}-t_{1}\right)^{\alpha} .
\end{aligned}
$$

As $t_{1} \rightarrow t_{2}$, the right-hand side of inequality (3.8) tends to zero and the convergence is independent of $u$ in $\Omega$, which means that $F_{1}(\Omega)$ is equicontinuous. The Arzela-Ascoli theorem implies that $F_{1}$ is compact.

Step 3. We show that $F_{1}$ is continuous.

Let $\left(u_{n}\right)$ be a sequence such that $u_{n} \rightarrow u$ in $\Omega$, we have

$$
\left|\left(F_{1} u_{n}\right)(t)-\left(F_{1} u\right)(t)\right| \leq \int_{0}^{t} \frac{(t-q s)^{(\alpha-1)}}{\Gamma_{q}(\alpha)}\left|h_{u_{n}}(s)-h_{u}(s)\right| d_{q} s,
$$


where $h_{u_{n}} \in C(J, \mathbb{R})$ be such that

$$
h_{u_{n}}(t)=f\left(t, u_{n}(t), h_{u_{n}}(t)\right) \text {. }
$$

Since $u_{n} \rightarrow u$ as $n \rightarrow \infty$ and $f$ is continuous function, we get

$$
h_{u_{n}}(t) \rightarrow h_{u}(t) \text { as } n \rightarrow \infty \text {. }
$$

Then, the Lebesgue dominated convergence theorem implies that

$$
\left|\left(F_{1} u_{n}\right)(t)-\left(F_{1} u\right)(t)\right| \rightarrow 0 \text { as } n \rightarrow \infty .
$$

This shows that $\left(F_{1} u_{n}\right)$ converges pointwise to $F_{1} u$ on $J$. Moreover, the sequence $\left(F_{1} u_{n}\right)$ is equicontinuous by a similar proof of Step 2. Therefore $\left(F_{1} u_{n}\right)$ converges uniformly to $F_{1} u$ and hence $F_{1}$ is continuous.

Step 4. We prove that $F_{2}: \Omega \rightarrow C(J, \mathbb{R})$ is a contraction mapping.

For all $u, v \in \Omega$ and $t \in J$, we have

$$
\begin{aligned}
\left|\left(F_{2} u\right)(t)-\left(F_{2} v\right)(t)\right| & =|g(u)-g(v)| \\
& \leq L_{g}\|u-v\|
\end{aligned}
$$

thus

$$
\left\|F_{2} u-F_{2} v\right\| \leq L_{g}\|u-v\|
$$

Hence, the operator $F_{2}$ is a contraction.

Clearly, all the hypotheses of the Krasnoselskii fixed point theorem are satisfied. Hence, there a fixed point $u \in \Omega$ such that $u=F_{1} u+F_{2} u$ which is a solution of the problem (1.1).

Example 3.1. We consider the following problem of implicit fractional $\frac{1}{2}$-difference equations

$$
\left\{\begin{array}{l}
D_{\frac{1}{2}}^{\frac{1}{2}} u(t)=\frac{1}{(\exp (t)+3)\left(1+|u(t)|+\left|{ }^{C} D_{\frac{1}{2}}^{\frac{1}{2}} u(t)\right|\right)}, t \in J=[0,1], \\
u(0)-\sum_{i=1}^{n} c_{i} u\left(t_{i}\right)=1,
\end{array}\right.
$$

where $T=1, \alpha=q=\frac{1}{2}, u_{0}=1,0<t_{1}<\ldots<t_{n}<1$ and $c_{i}, i=1, \ldots, n$ are positive constants with

$$
\sum_{i=1}^{n} c_{i} \leq \frac{1}{6}
$$


Set

$$
\begin{aligned}
f\left(t, u_{1}, v_{1}\right) & =\frac{1}{(\exp (t)+3)\left(1+\left|u_{1}\right|+\left|v_{1}\right|\right)}, t \in J, u_{1}, v_{1} \in \mathbb{R}, \\
g(u) & =-\sum_{i=1}^{n} c_{i} u\left(t_{i}\right), u \in C(J, \mathbb{R}) .
\end{aligned}
$$

For each $u_{1}, u_{2}, v_{1}, v_{2} \in \mathbb{R}$ and $t \in J$, we have

$$
\begin{aligned}
& \left|f\left(t, u_{1}, v_{1}\right)-f\left(t, u_{2}, v_{2}\right)\right| \\
& =\left|\frac{1}{(\exp (t)+3)}\left(\frac{1}{\left(1+\left|u_{1}\right|+\left|v_{1}\right|\right)}-\frac{1}{\left(1+\left|u_{2}\right|+\left|v_{2}\right|\right)}\right)\right| \\
& \leq \frac{\left|u_{1}-u_{2}\right|+\left|v_{1}-v_{2}\right|}{(\exp (t)+3)\left(1+\left|u_{1}\right|+\left|v_{1}\right|\right)\left(1+\left|u_{2}\right|+\left|v_{2}\right|\right)} \\
& \leq \frac{1}{4}\left(\left|u_{1}-u_{2}\right|+\left|v_{1}-v_{2}\right|\right)
\end{aligned}
$$

And for each $u, v \in C(J, \mathbb{R})$, we get

$$
\begin{aligned}
|g(u)-g(v)| & \leq \sum_{i=1}^{n} c_{i}\left|u\left(t_{i}\right)-v\left(t_{i}\right)\right| \\
& \leq \sum_{i=1}^{n} c_{i}\|u-v\| \leq \frac{1}{6}\|u-v\| .
\end{aligned}
$$

Hence, conditions (H1) and (H2) are satisfied with $L_{1}=L_{2}=\frac{1}{4}$ and $L_{g}=\frac{1}{6}$. The condition

$$
L_{g}+\frac{L_{1} T^{\alpha}}{\left(1-L_{2}\right) \Gamma_{q}(\alpha+1)}=\frac{1}{6}+\frac{\frac{1}{4}}{\left(1-\frac{1}{4}\right) \Gamma_{q}\left(\frac{3}{2}\right)} \simeq 0.49<1,
$$

is satisfied. It follows from Theorem 3.1 that the problem (1.1) has a unique solution on J.

\section{REFERENCES}

[1] S. Abbas, M. Benchohra, N. Laledj, Y. Zhou, Existence and Ulam stability for implicit fractional q-difference equations, Adv. Differ. Equ. 2019(1) (2019), 1-12.

[2] R. P. Agarwal, Certain fractional q-integrals and q-derivatives, Proc. Camb. Philos. Soc. 66(1) (1969), 365-370.

[3] B. Ahmad, S. K. Ntouyas, L. K. Purnaras, Existence results for nonlocal boundary value problems of nonlinear fractional q-difference equations, Adv. Differ. Equ. 2012(1) (2012), 1-14.

[4] W. A. Al-Salam, Some fractional q-integrals and q-derivatives, Proc. Edinb. Math. Soc. 15(2) (1966-1967), 135-140.

[5] A. Ardjouni, Positive solutions for nonlinear Hadamard fractional differential equations with integral boundary conditions, AIMS Math. 4(4) (2019), 1101-1113. 
[6] A. Ardjouni, A. Djoudi, Positive solutions for first-order nonlinear Caputo-Hadamard fractional relaxation differential equations, Kragujevac J. Math. 45(6) (2021), 897-908.

[7] A. Ardjouni, A. Djoudi, Initial-value problems for nonlinear hybrid implicit Caputo fractional differential equations, Malaya J. Mat. 7(2) (2019), 314-317.

[8] A. Ardjouni, A. Djoudi, Approximating solutions of nonlinear hybrid Caputo fractional integro-differential equations via Dhage iteration principle, Ural Math. J. 5(1) 2019, 3-12.

[9] A. Ardjouni, A. Djoudi, Existence and uniqueness of positive solutions for first-order nonlinear LiouvilleCaputo fractional differential equations, São Paulo J. Math. Sci. 14 (2020), 381-390.

[10] A. Bashir, S. Sivasundaram, Some existence results for fractional integro-differential equations with nonlocal conditions, Commun. Appl. Anal. 12(2) (2008), 107-112.

[11] M. Benchohra, S. Bouriah, M. A. Darwish, Nonlinear boundary value problem for implicit differential equations of fractional order in Banach spaces, Fixed Point Theory 18(2) 2017, 457-470.

[12] M. El-Shahed, F. M. Al-Askar, On the existence and uniqueness of solutions for q-fractional boundary value problem, Int. J. Math. Anal. 5 (2011), 1619-1630.

[13] S. Etemad, S. K. Ntouyas, B. Ahmad, Existence theory for a fractional q-integro-difference equation with q-integral boundary conditions of different orders, Mathematics 7(8) (2019), 659.

[14] R. A. C. Ferreira, Nontrivial solutions for fractional q-difference boundary value problems, Electron. J. Qual. Theory Differ. Equ. 2010 (2010), 70.

[15] M. Haoues, A. Ardjouni, A. Djoudi, Existence, interval of existence and uniqueness of solutions for nonlinear implicit Caputo fractional differential equations, Transylvanian J. Math. Mech. (TJMM) 10(1) (2018), 9-13.

[16] F. H. Jackson, On q-functions and a certain difference operator, Trans. Roy. Soc. Edinburgh 46 (1908), 253-281.

[17] F. H. Jackson, On q-definite integrals, Quart. J. Pure Appl. Math. 41 (1910), 193-203.

[18] V. Kac, P. Cheung, Quantum Calculus, Springer, New York, NY, USA, 2002.

[19] A. A. Kilbas, H. M. Srivastava and J. J. Trujillo, Theory and Applications of Fractional Differential Equations, Elsevier Science B. V., Amsterdam, 2006.

[20] K. D. Kucche, J. J. Nieto and V. Venktesh, Theory of nonlinear implicit fractional differential equations, Differ. Equ. Dyn. Syst. 28 (2020), 1-17.

[21] I. Podlubny, Fractional Differential Equations, Academic Press, San Diego, 1999.

[22] P. M. Rajkovic, S. Marinkovic, M. Stankovic, Fractional integrals and derivatives in q-calculus, Appl. Anal. Discr. Math. 1 (2007), 311-323.

[23] P. M. Rajkovic, S. Marinkovic, M. Stankovic, On q-analogues of Caputo derivative and Mittag-Leffler function, Fract. Cal. Appl. Anal. 10(4) (2007), 359-373.

[24] D. R. Smart, Fixed Point Theorems, Cambridge Tracts in Mathematics, Cambridge University Press, LondonNew York, 1974.

[25] F. Wang, Existence and uniqueness of solutions for a nonlinear fractional differential equation, J. Appl. Math. Comput. 39(1-2) (2012), 53-67. 
[26] W. Yang, Anti-periodic boundary value problems involving nonlinear fractional q-difference equations, Malaya J. Mat. 4(1) (2013), 107-114.

[27] Y. Zhao, H. Chen, Q. Zhang, Existence results for fractional q-difference equations with nonlocal q-integral boundary conditions, Adv. Differ. Equ. 2013 (2013), 48.

[28] Y. Zhou, J. R. Wang, L. Zhang, Basic Theory of Fractional Differential Equations, Second edition, World Scientific Publishing Co. Pte. Ltd., Hackensack, NJ, 2017. 